\title{
Estimation of Auroral Environment by Electrostatic Full-particle Simulations Modeling of REIMEI Satellite Observations
}

\author{
By Hiroko O. UEDA ${ }^{12)}$, Masaki OKADA ${ }^{3)}$, Hideyuki USUI ${ }^{4)}$, Takanobu MURANAKA ${ }^{1)}$ and Iku SHINOHARA ${ }^{1)}$ \\ 1) Japan Aerospace Exploration Agency, Sagamihara, Japan \\ 2) Japan Science and Technology Agency, CREST, Tokyo, Japan \\ ${ }^{3)}$ National Institute of Polar Research, Tokyo, Japan, \\ ${ }^{4)}$ Kyoto University, Uji, Japan \\ (Received May 2nd, 2008)
}

\begin{abstract}
A full particle-in-cell (PIC) plasma simulator with higher accuracy as well as reasonable performance for studying spacecraft-plasma interactions has been developed. It is applied to estimate characteristics of the onboard current monitors (CRM) for a small scientific satellite REIMEI in the polar orbit, because calibration is required for the observation data. Basic characteristics in typical plasma environments including effects of the satellite geometry are demonstrated.
\end{abstract}

Key Words: auroral environment, spacecraft charging, Langmuir probe, PIC simulation

\section{Nomenclature}

e :charge of electrons

$I_{e} \quad$ :electron current collected by a probe

$I_{e s} \quad$ :saturation level of $I_{e}$

$I_{+} \quad$ :ion current collected by a probe

$m_{e} \quad$ :mass of electrons

$m_{+} \quad$ :mass of ions

$n_{\rho} \quad$ :electron density

$n_{+} \quad$ :ion density

$S_{n} \quad$ :exposed area of a probe

$T_{\rho} \quad$ :electron temperature

$V_{n} \quad$ :bias voltage for a probe

$\kappa \quad$ :Boltzmann constant

$\lambda_{n} \quad$ :Debye length

\section{Introduction}

The small scientific satellite REIMEI launched on August 24, 2005, has continued the observation of auroral environment at the altitude of $610 \mathrm{~km}$ to $670 \mathrm{~km}$ in the sun-synchronous polar orbit ${ }^{1}$. It is equipped with an aurora camera $^{2)}$, two particle sensors for electrons and ions ${ }^{3)}$, a patch type single-probe so called Langmuir probe and two double-probes for the plasma-current monitors (CRM) designed for high-time/spatial resolution measurements. Theoretically, the background plasma parameters can reasonably be obtained from the probe characteristics over most of the polar regions. The data is expected to show changing of the plasma temperature during aurora particle precipitation, as statistical studies have suggested.
As the high energy particle impinging upon a satellite body, the floating potential of a piggy back satellite such like REIMEI easily moves according to the charging of the satellite. The LEO satellites also have to take into account the drift velocity of the satellite itself, because the satellite velocity is usually faster than the ion thermal velocity. More over in REIMEI satellite case, the aurora particles flow along the Earth's magnetic field which is orthogonal to the satellite's orbiting velocity. Although some chamber experiments for the newly developed probes are conducted, quantitative characteristics of CRM in the auroral environment including effects of the satellite geometry have not been obtained. A numerical simulation will be helpful to estimate the characteristics, if it could reproduce the current collection of the probe in the plasma environment.

For study of spacecraft-plasma interactions, higher accuracy as well as reasonable performance is required to numerical simulations. Achieving both requirements, we have developed the numerical space chamber for spacecraft in electrostatic environment based on full particle-in-cell (PIC) plasma code ${ }^{45)}$ optimized for parallel supercomputers. Multi-Utility Spacecraft Charging Analysis Tool $(\text { MUSCAT) })^{67)}$ developed by Kyusyu Institute of Technology and JAXA is another approach focusing on charging analysis for engineering aspect. The numerical spacecraft modeling techniques of MUSCAT is combined to our simulator, so that it enables us to study nonstationary electrostatic geospace environment with realistic spacecraft modeling. As the first application of the simulator, we have worked on interpretation of the observation data by REIMEI satellite.

In this paper, we present in the following section an overview of the simulator including accuracy tests measuring the characteristics of Langmuire probe in some chamber plasma parameters. 
In section 3, we describe the simulation model for REIMEI current monitors and results of the simulations. Firstly the basic characteristics of CRM in a thermal plasma are discussed, which is affected by the satellite body potential. Next the characteristics under the drifting satellite environment and also under the precipitating aurora electrons are shown.

In section 4, we summarize that the simulations can reproduce valuable information which hardly be given by chamber experiments.

\section{Method and Validation of the Simulator}

Our aim is to analyze interaction processes of a spacecraft with space environments precisely using the simulator as well as consequences of the interactions. We employ PIC method for pushing plasma particles, and the capacity matrix method to obtain floating potentials of any internal bodies such as a spacecraft, emphasizing self-consistency in the simulator. Poisson's equation is solved by the sine transform, therefore the plasma potential on the outer boundary of the simulation region is kept at zero.

We have tuned the simulation code to some distributed memory parallel computer systems to achieve desirable performance. The activity has been taken over the project of Geospace Environment Simulator (GES) development ${ }^{4)}$ in which the periodic electromagnetic PIC code achieved the parallelization ratio $99 \%$ on such systems.

In order to confirm validity of the simulations, we initially performed a simplified Langmuir probe model in a thermal plasma condition. Numerical parameters such as grid length, time step, and number of particles are given appropriately for accuracy. Figure 1 shows the I-V characteristic curve which is a plot of the electron current collected by a $7 \mathrm{~cm}$ cubic probe vs. bias voltage. The bias voltage is swept linearly from the floating potential of the probe. The potential distribution during the bias voltage being $0 \mathrm{~V}$ is shown in Fig. 2. The electron temperature is generally estimated ${ }^{8)}$ from the gradient of the curve of Fig. 1 as follows,

$$
\frac{d}{d V_{p}} \ln \left(I_{e}\right)=-\frac{e}{\kappa T_{e}}
$$

where $\kappa T_{e} / e$ is the electron temperature in the unit of $\mathrm{eV}$. The electron density for a plate probe is given from the equation.

$$
I_{e s}=e n_{e} S_{p} \sqrt{\frac{\kappa T_{e}}{2 \pi m_{e}}}
$$

For such a case that saturation level of the current is not visible, $I_{e s}$ is fundamentally set to the value at inflected point from the tangent line L1, that is the point where the bias voltage is equivalent to the plasma potential. On the other hand, the value at intersection of two tangent lines L1 and L2 is sometimes assigned. Table 1 shows the results of estimation and the ideal values as references. The temperature and the density given by $\mathrm{I}_{\mathrm{e} 1}$ are highly accurate, while an error of $20 \%$ is observed for the density by $\mathrm{I}_{\mathrm{e} 2}$. It suggests that the readout of the current causes considerable degree of error on the density estimation. We employ the latter as the saturation current in this study because of less artificial adjustment.

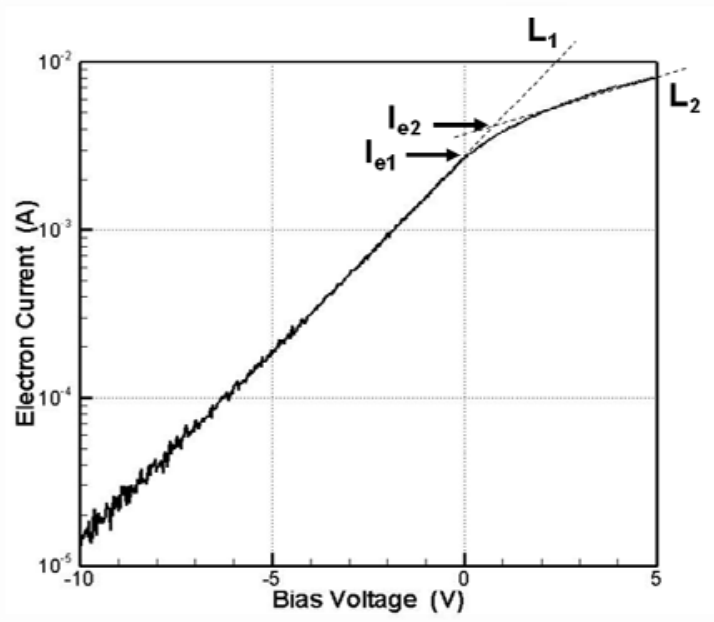

Fig. 1. The I-V characteristic curve of the Langmuir probe test simulation.

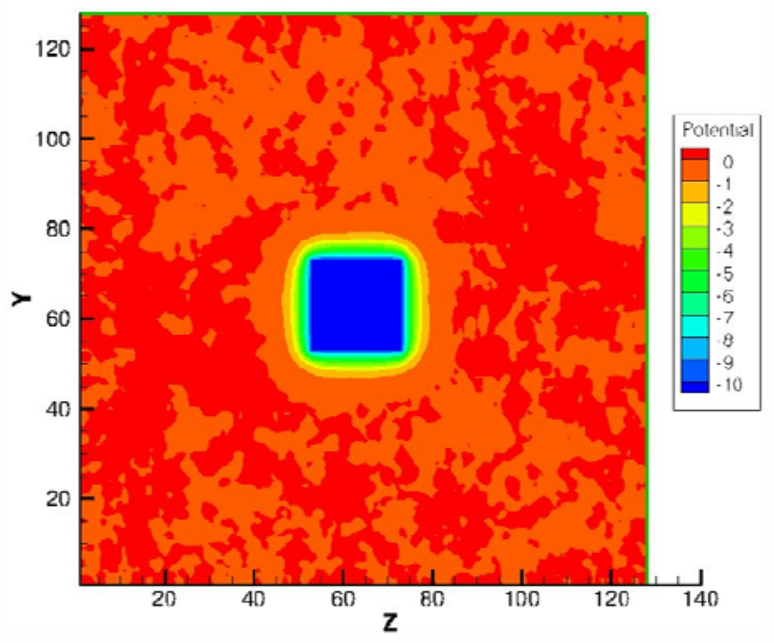

Fig. 2. The potential distribution at bias voltage $=0 \mathrm{~V}$. The grid length $\mathrm{dr}=1 / 2 \lambda_{\mathrm{D}}$, each side of the cubic internal probe is 20 times $\mathrm{dr}$.

Table 1. The estimation results of the Langmuir probe test.

\begin{tabular}{|c|c|c|c|}
\hline \multicolumn{2}{|c|}{} & $\begin{array}{c}\text { Temperature } \\
{[\mathrm{eV}]}\end{array}$ & $\begin{array}{c}\text { Density } \\
{\left[\mathrm{m}^{-3}\right]}\end{array}$ \\
\hline \multicolumn{2}{|c|}{ Set Value } & 2.0 & $3.0 \times 10^{12}$ \\
\hline \multirow{2}{*}{ Estimation } & $\mathrm{I}_{\mathrm{e} 1}$ & 1.95 & $3.0 \times 10^{12}$ \\
\cline { 2 - 2 } & $\mathrm{I}_{\mathrm{e} 2}$ & & $3.6 \times 10^{12}$ \\
\cline { 4 - 5 }
\end{tabular}




\section{Simulations for REIMEI satellite}

\subsection{Simulation Model}

We have modeled the satellite and plasmas in this study as close as to the real parameters. Figure 3 illustrates the simulation model of REIMEI satellite. It consists of a $50 \mathrm{~cm}$ cubic main body, solar panels and solar reflectors on the both paddles. Two sets of double-probes named as DP-1 and DP-2 in this paper, are insulated from the body. A bias voltage of single-probe named as SP is applied by reference to the body potential during sweep. Each patch type probe of CRM is $5 \mathrm{~cm}$ squared which is smallest grid size though the real size is 2.2 $\mathrm{cm}$. The difference should not affect the estimations of temperature and density given by Eq. (1) and (2), whereas it should to the probe current. The sweep period is about $1.5 \mathrm{~ms}$ which is more than 10 times longer than ion plasma period in the simulation while much shorter than $320 \mathrm{~ms}$ for the real CRM. All the materials including the solar panels are assumed as a conductor. Floating potential of the satellite is not exact in some cases as a result. For a typical attitude in polar region, it can be assumed that the satellite drifts toward $\mathrm{z}$ direction and

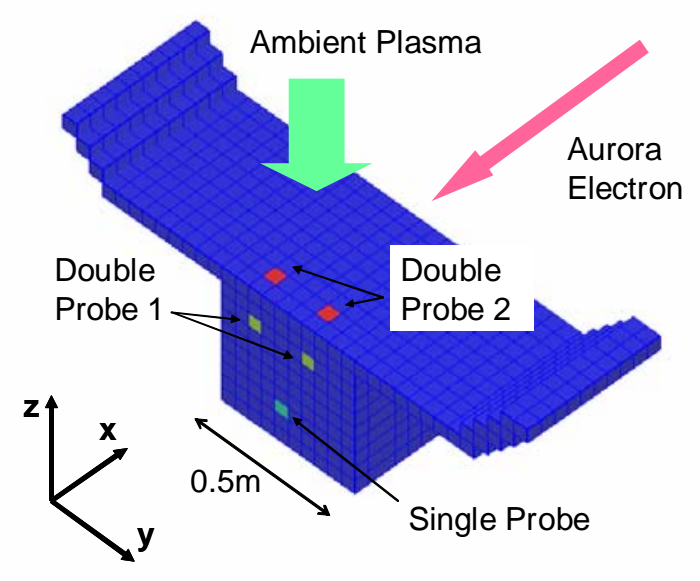

Fig. 3. Schematic illustration of REIMEI simulation model.

Table 2. Parameters for REIMEI simulations.

\begin{tabular}{|c|c|c|}
\hline \multirow{2}{*}{$\begin{array}{l}\text { Ambient } \\
\text { Plasma }\end{array}$} & Density & $2.0 \times 10^{9}\left[\mathrm{~m}^{-3}\right]$ \\
\hline & Temperature & $0.20[\mathrm{eV}]$ \\
\hline \multirow{3}{*}{$\begin{array}{l}\text { Aurora } \\
\text { Electron }\end{array}$} & Density & $1.5 \times 10^{7}\left[\mathrm{~m}^{-3}\right]$ \\
\hline & Temperature & $750[\mathrm{keV}]$ \\
\hline & Drift Energy & $7.5[\mathrm{keV}]$ \\
\hline \multicolumn{2}{|c|}{ Satellite Drift Velocity } & $8.0[\mathrm{~km} / \mathrm{s}]$ \\
\hline \multirow[t]{3}{*}{$\begin{array}{l}\text { Simulation } \\
\text { System }\end{array}$} & $\begin{array}{l}\text { Number of Grids } \\
\mathrm{nx} \times \mathrm{ny} \times \mathrm{nz}\end{array}$ & $128 \times 128 \times 128$ \\
\hline & $d x=d y=d z$ & $\begin{array}{c}0.05[\mathrm{~m}] \\
=1 / 2 \text { Debye length }\end{array}$ \\
\hline & $\mathrm{dt}$ & $5.0 \mathrm{e}^{-8}[\mathrm{~s}]$ \\
\hline
\end{tabular}

aurora electrons mostly precipitate to $-\mathrm{x}$ direction in Fig. 3. Parameters for the simulations are shown in Table 2. The grid length is $5 \mathrm{~cm}$ which is about a half of the Debye length of the ambient ionospheric plasma.

Dynamics of high energy aurora electrons are treated in the manner of particle tracking with 160 times shorter time step in the electric field only formed by the ambient plasma, because the density is much less than the ambient. Injection current into the satellite by the particle is sufficiently accurate.

We can monitor time histories of the satellite and space potentials, the configuration of the plasma sheath, and each probe current during sweeping the bias voltage.

\subsection{Basic characteristics of the single-probe (SP) in thermal plasma}

The collected currents of CRMs attached on the surface of the satellite are concerned about affection by the satellite body with large surface area. For the purpose of clearing the affection, we introduced in the simulations a temporal single-probe named TP which is assumed $5 \mathrm{~cm}$ cube far from the satellite.

We first measured each current of SP and TP in the thermal plasma during sweep biased to the body potential. Figures 4(a) and (b) show the potential and the electron density distributions, respectively, at bias voltage being $+1 \mathrm{~V}$. The electron density around the satellite is less than the background for the negatively charged body. The I-V characteristic curves for both probes are plotted in Fig. 5. The lines indicate total probe current including ion current. It is equivalent for SP to observed data on the satellite. The symbols indicate the electron currents. It took about $2 \mathrm{~ms}$ for one sweep in which the range of bias voltage is $-4 \mathrm{~V}$ to $4 \mathrm{~V}$. The estimations of electron temperature and density in the manner mentioned in the section 2 are shown in Table 3. The exposed area of the cubic TP is assumed as 6 times larger than SP. The ion density is given by the following equation.

$$
I_{+}=e n_{+} S_{p} \sqrt{\frac{\kappa T_{e}}{m_{+}}}
$$

The probe current at bias voltage being $-4 \mathrm{~V}$ is used as $I_{+}$and the estimated electron temperature from the I-V curve as $T_{e}$.

The electron temperature derived from TP electron current is almost accurate, and an error of $15 \%$ on the density is within the margin for readout. The temperature from the probe current is lower than that from the electron current by $25 \%$, and, as a result, higher density is given. It suggests that observed probe current without correction of the ion effect includes negligible error.

The temperatures from SP electron current and probe current in comparison to TP are much higher, respectively, and densities are lower as shown in Table 3. A possible cause is satellite body around SP which is charged in the range of -0.55 $\mathrm{V}$ to $-0.75 \mathrm{~V}$ during the sweep. Figure 6 shows speed distribution functions of the background electrons selected randomly, of electrons that come into SP and TP during the bias voltage being $0 \mathrm{~V}$ to $1 \mathrm{~V}$, respectively. The distributions 
suggest that a part of low energy electrons of TP cannot come inside SP because of the negatively charged body.

\section{(a) Potential in Thermal Plasma}

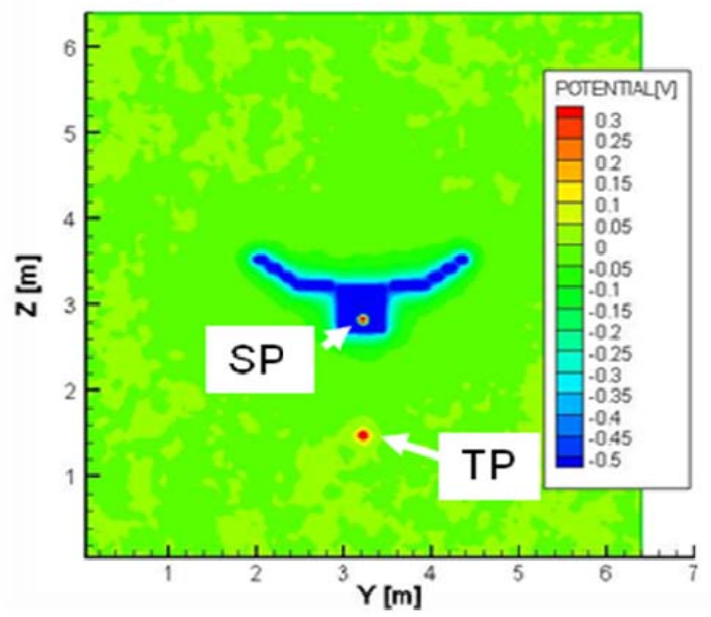

(b) Electron Density in Thermal Plasma

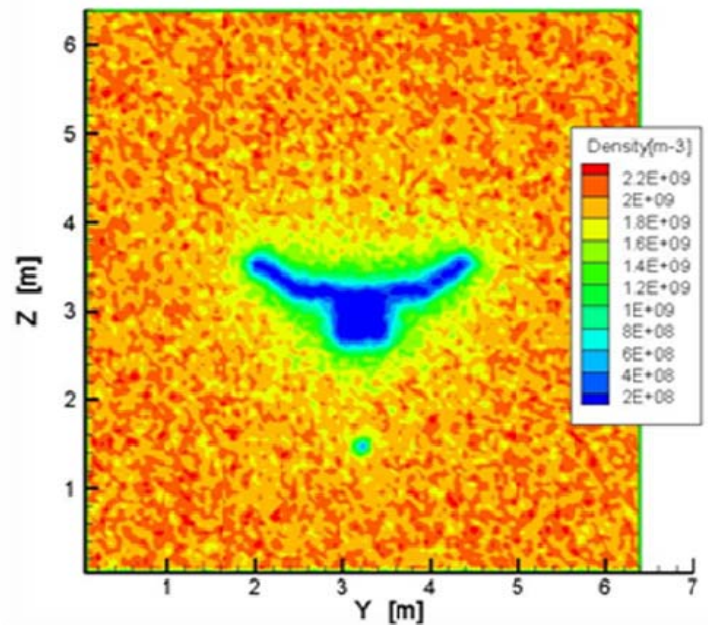

Fig. 4. The space distributions of (a)potential, and (b)charge density at bias voltage of the single-probes being $1 \mathrm{~V}$ in the thermal plasma.

Table 3. The estimation results from REIMEI single-probe and the temporal probe in the thermal plasma.

\begin{tabular}{|l|c|c|c|c|}
\hline \multicolumn{2}{|l|}{} & $\mathrm{kT}_{\mathrm{e}} / \mathrm{e}[\mathrm{eV}]$ & $\mathrm{n}_{\mathrm{e}}\left[\mathrm{m}^{-3}\right]$ & $\mathrm{n}_{+}\left[\mathrm{m}^{-3}\right]$ \\
\hline \multirow{2}{*}{ Set Value } & 0.20 & $2.0 \times 10^{9}$ & $2.0 \times 10^{9}$ \\
\hline \multirow{2}{*}{$\mathrm{TP}$} & Electron Cur. & 0.21 & $2.3 \times 10^{9}$ & --- \\
\cline { 2 - 5 } & Probe Cur. & 0.15 & $2.5 \times 10^{9}$ & $7.9 \times 10^{9}$ \\
\hline \multirow{2}{*}{$\mathrm{SP}$} & Electron Cur. & 0.34 & $1.9 \times 10^{9}$ & --- \\
\cline { 2 - 5 } & Probe Cur. & 0.21 & $2.1 \times 10^{9}$ & $1.3 \times 10^{10}$ \\
\hline
\end{tabular}

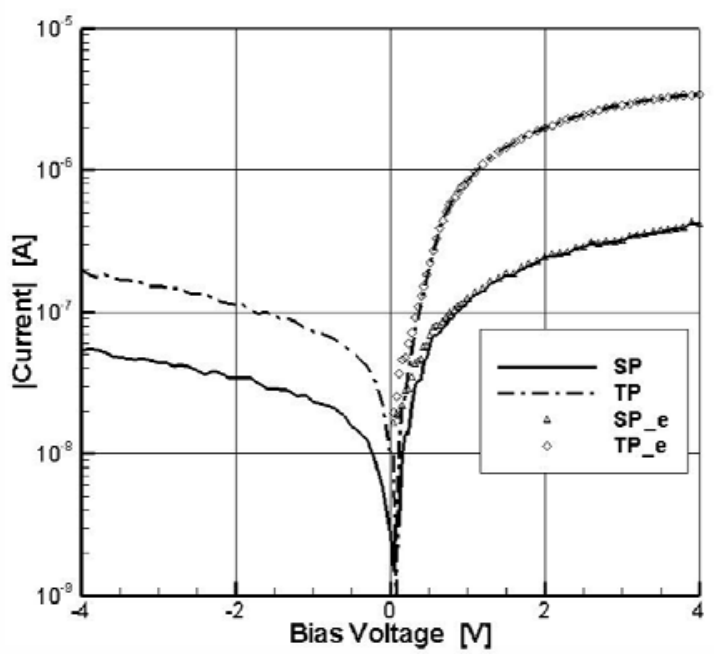

Fig. 5. The I-V characteristic curves of REIMEI single-probe and the temporal probe in the thermal plasma. The each line indicates total probe current including ion current, and the symbol is the electron current.

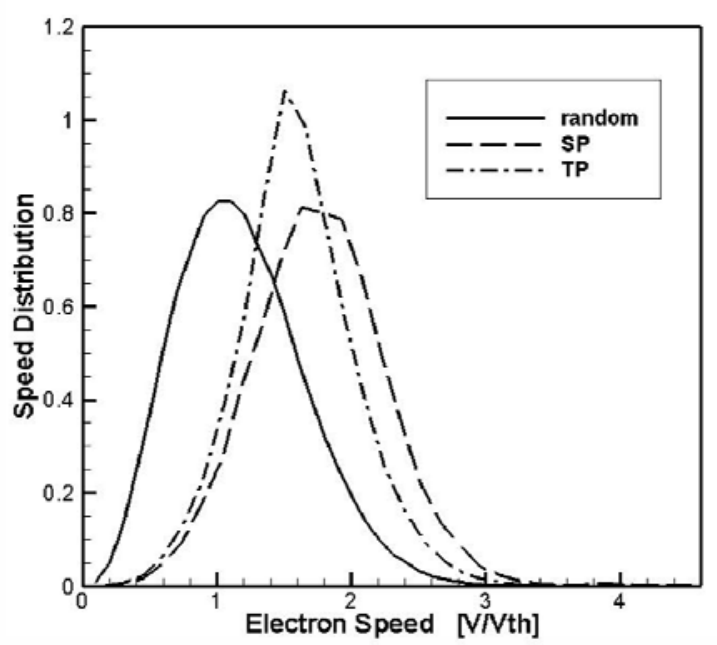

Fig. 6. The speed distribution functions of background electrons, of electrons coming inside SP and TP.

\subsection{Characteristics in drift plasma}

We take the effect of drifting satellite into the simulation conditions. The drift velocity is comparable to the ion thermal velocity. The direction of drift is perpendicular to the normal of SP and DP-1, that is the normal direction of DP-2. The lines of Fig. 7 show I-V curves of those probe currents in the drift plasma. The symbols are those in the thermal condition. It is obvious the ion current such for SP around floating potential as well as in minus voltage is lower than that in the thermal plasma. The estimation of electron temperature results in more accurate as shown in Table 4 . The body potential which is about $0.01 \mathrm{~V}$ higher than that in the thermal condition is regard 
as a cause.

The ion currents of DPs are basically high compared with that of SP because of the difference of the probe potential. In addition for DP-2, the drift ions are directly come inside. By contrast, the electron currents are affected little by the drift. The temperature and electron density estimated from DPs are not far from the set values. The electron currents are still useful for the estimation in the drift plasma, while the ion currents are not adequate.

Fig. 7. The I-V characteristic curves of REIMEI single-probe and the

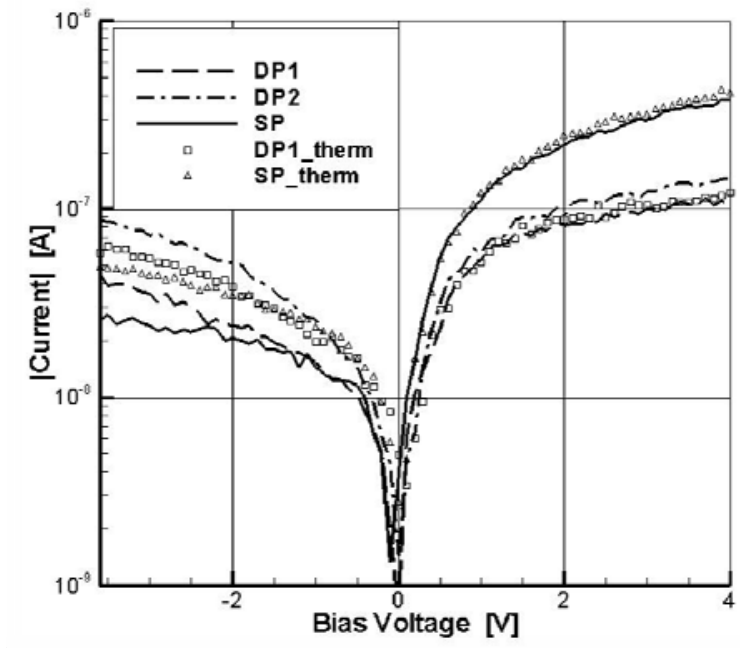

double-probes. The lines indicate probe current in the drift plasma, and the symbols in the thermal condition.

Table 4. The estimation results from REIMEI single-probe and the double-probes in the drift plasma.

\begin{tabular}{|c|c|c|c|}
\hline & $\mathrm{kT}_{\mathrm{e}} / \mathrm{e}[\mathrm{eV}]$ & $\mathrm{n}_{\mathrm{e}}\left[\mathrm{m}^{-3}\right]$ \\
\hline \multicolumn{2}{|c|}{ Set Value } & 0.2 & $2.0 \times 10^{9}$ \\
\hline \multirow{3}{*}{$\begin{array}{l}\text { Estim } \\
\text { ation }\end{array}$} & SP & 0.25 & $2.1 \times 10^{9}$ \\
\hline & DP-1 & 0.31 & $2.0 \times 10^{9}$ \\
\hline & DP-2 & 0.21 & $2.8 \times 10^{9}$ \\
\hline
\end{tabular}

\subsection{Simulation in precipitation of high energy electrons}

Here we only show a primary behavior of the satellite in the precipitating high energy electrons. The time histories of floating potential of the satellite, as well as inflow currents into the satellite by ions and by aurora electrons are plotted in Fig. 8. It is clear the body potential rapidly become out of the bias voltage. A large error will be inferred in such a case for the estimation of the plasma parameter using the probe data. For calibration of observed data by CRM, however, it is necessary to determine actual body potential. A few additional elements such as photo electrons and dielectrics as the cover glasses on the solar panels must be taken account in the simulation model. A succession of simulations including those effects is the next step.

\section{Summary and future work}

We initially showed high accuracy of the simulator for measuring the I-V characteristics of Langmuire probe. At the same time, the readout error of the current is considerable degree, at least $20 \%$, on the density estimation. The probe current without correction of the ion effect causes the error of $25 \%$ in the condition of typical low-earth-orbit plasma.

To clear the affection of the satellite body to the collected current of the single-probe (SP), we introduced the temporal single-probe (TP) located far from the satellite. The simulation suggested that a part of low energy electrons can not come into SP because of the negatively charged body, therefore the temperature and density given by SP data tends to be higher and lower, respectively, than those by TP. The effects of drifting satellite were also discussed. The electron current is still useful for the estimation compared with the ion current in the drift plasma.

It was demonstrated that the satellite potential rapidly become out of the bias voltage in the typical auroral environment. For calibration of CRM observation data, reproducing the actual body potential in the simulations is very important. Additional effects such as emission of photo electrons and dielectric materials have some impact on the body potential. We are ready to introduce the functions in the simulator. A qualitative data map for the calibration shall be obtained in our following work.

As mentioned above, investigations of detailed interactions between the small satellite and the high-density plasma are possible for our current simulator. However it still be difficult to apply for larger satellites which require larger number of

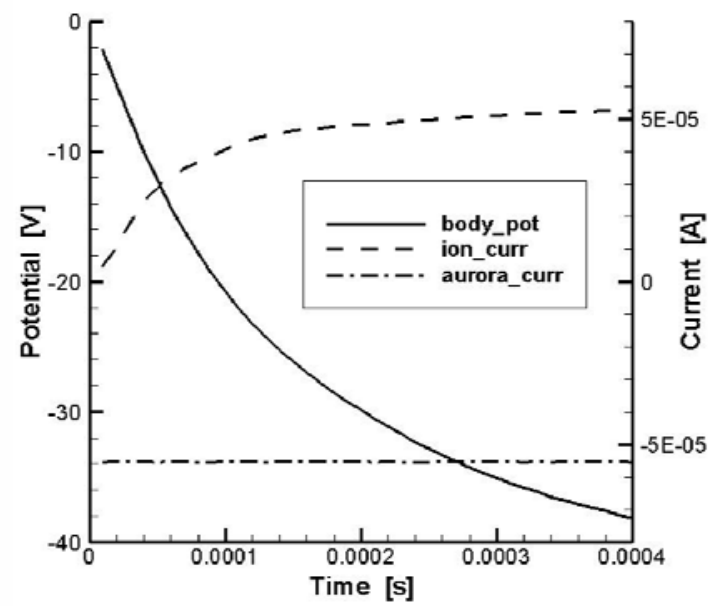

Fig. 8. The time histories of floating potential of REIMEI, and inflow currents by ions and by aurora electrons into the satellite. 
simulation grids because of performance of current super computers. We have improved the simulator for a resolution in which nested grids can be adopted to save drastically the computer resources.

\section{References}

1) H. Saito, T. Mizuno, K. Tanaka, Y. Sone, S. Fukuda, S. Sakai, N. Okuizumi, M. Mita, Y. Fukushima,M. Hirahara, K. Asamura, T. Sakanoi, A. Miura, T. Ikenaga, and Y. Masumoto: AN-OVERVIEW AND INITIAL IN-ORBIT STATUS OF "INDEX" SATELLITE, $56^{\text {th }}$ International Astronautical Conference, (2005) IAC-05-B5.6.B.05.

2) T. Sakanoi,, S. Okano, Y. Obuchi, T. Kobayashi, M. Ejiri, K. Asamura, and M. Hirahara: Development of the multi-spectral auroral camera onboard the INDEX satellite, Adv. Space Res. 32 (2003), pp.379-384.

3) K. Asamura,, D. Tsujita, H. Tanaka, Y. Saito, T. Mukai, and M. Hirahara: Auroral particle instrument onboard the INDEX satellite, Adv. Space Res., 32 (2003), pp.375-378.

4) M. Okada, H. Usui, Y. Omura, H. O. Ueda, T. Murata and T. Sugiyama: Spacecraft Plasma Environment Analysis via Large Scale 3D Plasma particle Simulation, ISHPC 2005 and ALPS 2006, LNCS 4759, Springer-Verlag Berlin Heidelberg, 2008, pp.383-392.

5) H. Usui, Y. Miyake, M. Okada, Y. Omura, T. Sugiyama, K. T. Murata, D. Matsuoka, H. O. Ueda: Development and Application of Geospace Environment Simulator for the Analysis of Spacecraft-plasma Interactions, IEEE Trans. Plasma Science, Vol.34, No.5 (2006), pp.2094-2102.

6) T. Muranaka, S. Hosoda, S. Hatta, J. Kim, K. Ikeda, M. Cho, H. Ueda, K. Koga, T. Goka: Final version of Multi-Utility Spacecraft Charging Analysis Tool (MUSCAT), IEEE Trans. on Plasma Science, Vol.35, No.5(2008), pp.2336-2349.

7) S. Hosoda, T. Muranaka, H. Ueda, K. Koga, T. Goka, H. Kuninaka, S. Hatta, J. Kim, N. Kurahara, M. Cho: Laboratory experiments for code validation of Multi-Utility Spacecraft Charging Analysis Tool (MUSCAT), IEEE Trans. on Plasma Science, Vol.35, No.5(2008), pp.2350-2359.

8) H. M. Mott-Smith and I. Langmuir: The Theory of Collectors in Gaseous Discharges, Phys. Rev., 28 (1926), pp.727-763. 